\title{
Special Issue on Motion Strategies for Underactuated Robotic Vehicles in the Presence of Constraints
}

\author{
Maciej Marcin Michałek • Andrea L'Afflitto • \\ Takateru Urakubo
}

Received: 1 May 2017 / Accepted: 8 May 2017 / Published online: 24 May 2017

(C) The Author(s) 2017. This article is an open access publication

\section{Introduction}

This special issue presents new solutions to theoretical and practical problems in motion planning and control of constrained, underactuated robotic systems. The increasing demand of autonomous robots in numerous and diverse applications, such as transportation, manufacturing, services, and defense, lead to a proliferation of methods which improve, extend, and complement existing motion planning and control strategies for multipurpose unmanned and robotic vehicles. This special issue provides a selection of approaches to these challenging problems.

M. M. Michałek ( $\square)$

Poznan University of Technology (PUT), Institute

of Automation and Robotics, Piotrowo 3A, 60-965

Poznań, Poland

e-mail: maciej.michalek@put.poznan.pl

\author{
A. L'Afflitto \\ School of Aerospace and Mechanical Engineering, \\ The University of Oklahoma, 865 Asp Ave., \\ Norman, OK 73019, USA \\ e-mail: a.lafflitto@ou.edu \\ T. Urakubo \\ Graduate School of System Informatics, Kobe University, \\ 1-1 Rokkodai-cho, Nada-ku, Kobe 657-8501, Japan \\ e-mail: t.urakubo@silver.kobe-u.ac.jp
}

Both motion planning and motion control are essential in robotic systems design for their roles in determining and executing the robots' tasks. However, designing the motion planning and control systems involved in advanced applications of autonomous robots is complicated not only for multi-agent systems, but also for a single vehicle. Indeed, in most cases of practical interest the robotic vehicles work in conditions of underactuation where the number of available control inputs is smaller than the number of degrees-of-freedom or smaller than the number of controlled outputs. Moreover, the complexity of planning and actuation strategies substantially increases due to the presence of obstacles in a robot workspace, mechanical limitations of the robots structure, saturation of control inputs, limitations of the on-board power, restrictions imposed on the task execution time, and limitations in the sensing and communication capabilities. Finding a solution to so complex problems becomes a serious challenge, especially when trying to provide strong guarantees concerning the stability, performance, and practical feasibility/applicability of a resultant motion strategy under relatively weak assumptions which could be satisfied in practice.

The fourteen papers collected in this special issue display a selection of the latest motion planning and control strategies, discuss their applicability to 
numerous problems, and highlight new challenges and future research directions in these fields.

\section{Special Issue Content}

Numerous scholars, who perform research in control theory, computing sciences, and robotics, contributed to this special issue. After a thorough peer-review process, 14 papers have been accepted for publication out of a pool of 24 competitive submissions initially received. Accepted manuscripts, which address a wide spectrum of topics concerning various types of vehicles, motion tasks, and constraints, can be grouped in three broad categories: 1) those addressing novel motion planning policies, 2) those concerning new (feedback) control laws, and 3) those combining novel planning and control algorithms into a coherent overall motion strategy.

Among the papers concerning motion planning strategies, we present the work by Ladosz, Oh, and Chen, wherein the optimal path for dynamically constrained unmanned aerial vehicles (UAVs), which are subject to communication constraints in a complex urban environment, is computed using nonlinear model predictive control. Nguyen, Recchiuto, and Sgorbissa propose a computationally efficient path planning method for multi-rotor UAVs in the presence of obstacles, wherein the vehicles path is defined as the intersection of suitably chosen surfaces. L'Afflitto and Haddad provide higher-order necessary conditions to solve singular and non-singular, abnormal optimal trajectory planning problems involving multi-body systems subject to holonomic and nonholonomic constraints. Neto, Macharet, and Campos propose a real-time motion planning strategy for groups of heterogeneous and underactuated robots subject to disturbances and uncertainties in cluttered constrained environments, which is based on the Rapidly-exploring Pseudo-random Tree approach. Finally, Casbeer, Garcia, and Pachter develop a differential game framework to address the optimal trajectory planning problem for a system comprised of UAV, an attacking missile, and a defending missile, wherein the defender is aimed at the attacker while the UAV is escaping.
In the second group of works, Zhao, Wang, Zhang, and Shen propose a path following controller for externally perturbed fixed-wing UAVs with input constraints, wherein the stability of the system for a curved desired path is shown by using the control Lyapunov function. Urakubo provides the stability analysis of equilibria when the potential field method is applied to nonholonomic systems, and designs a feedback control law for an underwater vehicle among obstacles. Cichella, Marinho, Stipanović, Hovakimyan, Kaminer, and Trujillo deal with the collision avoidance problem between UAVs and propose a feedback law that requires only the line-of-sight angle information; the Lyapunov-based analysis reveals the conditions under which the avoidance is theoretically guaranteed. Siravuru, Viswanathan, Sreenath, and Sanyal propose a new reduced-order model, called the Reaction Mass Biped (RMB), for bipedal locomotion, and design a controller for walking along straight and curved paths, wherein a discrete model of RMB is also developed to obtain accurate simulation results. Ziaeefard, Page, Pinar, and Mahmoudian present a control strategy for turning motion of an internally actuated underwater glider that has a novel roll mechanism, and demonstrate by experimental results that the control law improves maneuverability of the glider. Finally, Michałek and Gawron propose a path following controller for a nonholonomic unicycle by extending the Vector-Field-Orientation (VFO) method, wherein the desired path is expressed as a level curve of a function of a vehicle position. The authors provide formal conditions which guarantee satisfaction of the positional constraints imposed on a transient behavior of the controlled vehicle.

In the third group, Lopez-Padilla, Murrieta-Cid, Becerra, Laguna, and LaValle propose an optimal navigation strategy for differentially driven wheeled robots resulting in the shortest collision-free path. The robots considered in this paper move in a polygonal environment, whose geometric representation is partly known, and rely on the on-board sensors to account for the presence of obstacles. Viswanathan, Sanyal, and Samiei present an integrated guidance and feedback control strategy for rigid body vehicles with four control inputs moving in a three-dimensional space through a set of desired waypoints. This approach 
accounts for limitations of the vehicle's control inputs and generates at least twice differentiable robot trajectories. Finally, Gawron and Michałek introduce the combined VFO-controller-driven motion planning and feedback control strategy devised for unicycle-like mobile robots moving in the polygonal worlds with a limited motion curvature imposed.

We believe that this special issue will be inspiring and useful to the readers in their engineering-scientific research and applications.

\section{The Guest Editors,}

\section{Maciej M. Michałek Andrea L'Afflitto Takateru Urakubo}

\begin{abstract}
Acknowledgments We would like to thank Professor Kimon Valavanis, Editor-In-Chief of the Journal of Intelligent \& Robotic Systems, for the opportunity of organizing this special issue and for his support in this initiative. We also kindly thank Ms. Marie Veth Chua from the Springer team for her help in the administrative work. Lastly, we thank all the Authors for their interesting contributions and express our gratitude to all Reviewers for their time and efforts devoted to the extensive review process.
\end{abstract}

Open Access This article is distributed under the terms of the Creative Commons Attribution 4.0 International License (http:// creativecommons.org/licenses/by/4.0/), which permits unrestricted use, distribution, and reproduction in any medium, provided you give appropriate credit to the original author(s) and the source, provide a link to the Creative Commons license, and indicate if changes were made.
Maciej Marcin Michałek received the M.Sc., Ph.D., and D.Sc. (Habilitation) degrees in automation and robotics from the Poznan University of Technology (PUT), Poznań, Poland, in the years 2001, 2006, and 2015, respectively. He is currently an assistant professor with the Institute of Automation and Robotics, Faculty of Computing, PUT. His current research interests include problems of control design and control applications for nonholonomic systems, in particular, for mobile robots and $\mathrm{N}$-trailer vehicles, and for systems with uncertain and nonminimum-phase dynamics. Dr. Michałek has been a member of the IEEE since 2009. He serves as an Editorial Board Member of the Journal of Intelligent and Robotic Systems.

Andrea L'Afflitto received the B.S. and M.S. degrees in aerospace engineering from the University of Napoli, Italy, "Federico II," in 2004 and 2006, respectively, the M.S. degree in mathematics from Virginia Tech in 2011, and the Ph.D. degree in aerospace engineering from Georgia Tech in 2015. Since 2015, he has been with the School of Aerospace and Mechanical Engineering of the University of Oklahoma as an assistant professor. Dr. L'Afflitto's research focusses on optimal control, differential games, and nonlinear robust control and estimation with applications to underactuated mechanical systems, such as multi-rotor aircraft. Dr. L'Afflitto is the author of a monograph on flight dynamics and control, one book chapter, and 26 journal and conference papers.

Takateru Urakubo received the B.E., M.E. and Ph.D. degrees in aeronautics and astronautics from Kyoto University, Kyoto, Japan, in 1996, 1998 and 2001, respectively. He joined Kobe University, Kobe, Japan, as an assistant professor in 2001, where he is currently an associate professor with the Graduate School of System Informatics. From 2007 to 2009, he was a visiting research scientist at Carnegie Mellon University, PA, USA. His current research interests include nonlinear dynamical systems, nonlinear control theory and autonomous robots. 